\title{
Uso e Impacto de los Modelos nD como Herramienta para la Dirección de Proyectos en la Industria de la Arquitectura, Ingeniería y Construcción
}

\author{
Rodrigo F. Herrera ${ }^{1,2}$, Felipe C. Muñoz-La Rivera ${ }^{2}$, Claudio F. Vargas ${ }^{2}$ y Madelinne M. Antio ${ }^{2}$ \\ (1) Pontificia Universidad Católica de Chile, Departamento de Ingeniería y Gestión de la Construcción, \\ Vicuña Mackenna 4860, Macul, Santiago-Chile. \\ (2) Pontificia Universidad Católica de Valparaíso, Escuela de Ingeniería Civil, Avenida Brasil 2147, \\ Valparaíso-Chile. (e-mail: rodrigo.herrera@pucv.cl, fmunozlarivera@gmail.com, \\ claudio.vargas.aguila@gmail.com, madelinne.antio@gmail.com
}

Recibido Ene. 23, 2017; Aceptado Mar. 16, 2017; Versión final Abr. 12, 2017, Publicado Ago. 2017

\begin{abstract}
Resumen
El principal objetivo de esta investigación es presentar evidencia del uso e impacto de modelos $\mathrm{nD}$ como herramienta para la dirección de proyectos en la industria de la arquitectura, la ingeniería y la construcción, para lo cual se ha realizado una revisión bibliográfica sobre el tema. La industria de la arquitectura, ingeniería y construcción ha sufrido cambios, innovaciones y avances en pos de optimizar proyectos pertenecientes al rubro. En este contexto, las tecnologías de información se han ido incorporando al desarrollo de este tipo de obras. Sin embargo, se desconoce el uso e impacto real que estas herramientas entregan en cada una de las áreas de conocimiento en la dirección de proyectos. Dentro de los resultados principales se tiene que al implementar estos modelos se produce un impacto directo e indirecto en la gran mayoría de los procesos de dirección de proyectos y con mayor énfasis en las áreas tiempo, costos e clientes.
\end{abstract}

\section{Use and Impact of $\mathrm{nD}$ Models as a Tool for Project Management in the Architecture, Engineering and Construction Industry}

\begin{abstract}
The main objective of this research is to present evidence on the use and impact of $\mathrm{nD}$ models as a tool for project management in the architecture, engineering and construction industry, for which a bibliographic review on the subject has been carried out. The architecture, engineering and construction industry has undergone changes, innovations and advances to optimize projects belonging to the field. In this context, information technologies have been incorporated into the development of this type of works. However, the use and real impact that these tools contribute to each of the areas of knowledge in project management is unknown. Among the main results, the implementation of these models has a direct e indirect impact on the great majority of project management processes, with a greater emphasis on time, cost and stakeholders.
\end{abstract}

Keywords: $n D$ models, project management, $A E C$ industry, project costs 


\section{INTRODUCCIÓN}

La gestión de proyectos en la industria de la Arquitectura, Ingeniería y Construcción (AIC) representa particularmente un desafío en comparación a otras áreas productivas, dada la temporalidad de las mismas, lo marcadas y separadas que son sus diversas áreas (dentro del "proceso") entre otros factores difíciles de controlar (Fulford y Standing, 2014). En este sentido, evidentes son las etapas de pre-diseño, diseño, ejecución en terreno y monitoreo-control de cada una de ellas. Las tecnologías de la información en la industria de la AIC representan herramientas potentes para facilitar la dirección, planificación y control de proyectos de esta área, registrándose un importante aumento en su uso en la última década (Mourgues y Fischer, 2001). Sin perjuicio de lo anterior, se desconoce el real uso e impacto que estas herramientas tienen en las distintas áreas de conocimiento propuestas por el Project Management Institute (PMI) en la dirección de proyectos.

Las tecnologías de la información en la industria de la AIC vienen siendo una herramienta potente para facilitar la dirección, planificación y control de proyectos de esta área, registrándose un importante aumento en su uso en la última década (Mourgues y Fischer, 2001). En cuanto a estas nuevas tecnologías, aun cuando éstas proponen una forma de trabajo mucho más eficiente que la tradicional, esta eficiencia no es aplicable de manera inmediata, dado la amplia gama de factores que rigen el funcionamiento de un proyecto, por lo cual, las nuevas tecnologías deben encajar en el ecosistema existente, o esperar que el ecosistema se adapte, proceso mucho más lento (Sánchez y Valderrama, 2013). Sin perjuicio de lo anterior, se desconoce el real uso e impacto que estas herramientas tienen en las distintas áreas de conocimiento propuestas por el Project Management Institute (PMI) en la dirección de proyectos. A lo largo de los años la representación del trabajo en la construcción ha ido evolucionado desde planos 2D en papel hasta los modelos multidimensionales o $\mathrm{nD}$. Los modelos $\mathrm{nD}$ son representaciones virtuales que comienzan con las representaciones virtuales en tres dimensiones y que luego se le agregan otras variables, tales como, tiempo (4D), costo (5D) y otros análisis (nD). Éstos tienen por objetivo representar y visualizar el proyecto global, permitiendo mejorar el proceso de toma de decisiones durante las distintas etapas de un proyecto. Muchas veces, se confunden los modelos $\mathrm{nD}$ con los modelos BIM (Building Information Model), sin embargo, un modelo $\mathrm{nD}$ no necesariamente es BIM ni tampoco al revés. BIM está enfocado más en el modelo en sí, mientras que nD es una herramienta para análisis (Ding et al., 2014)

Ante la potencial masificación de los modelos $\mathrm{nD}$ se hace necesario conocer la utilización y el impacto de dichas herramientas en la dirección de proyectos y por ende, en las obras donde se implemente (Ding et al., 2014). Es con la información anterior que podrán enfocarse los esfuerzos en potenciar aquellas áreas donde se han obtenidos buenos resultados y a su vez comenzar la investigación en otras de los cuales los antecedentes sean más escasos. Por esta razón el objetivo de este artículo es presentar evidencia del uso e impacto de la utilización de modelos $\mathrm{nD}$ en las áreas de conocimiento propuestas por el PMI, como herramienta en la dirección de proyectos en la industria de la AIC. Además, se busca identificar si la utilización de modelos nD logran aumentar la integración en los proyectos de la industria de la AIC.

Para cumplir con los objetivos, se realizó una revisión bibliográfica de 29 artículos científicos donde se presenta evidencia del uso e impacto de los modelos $\mathrm{nD}$ en la gestión de proyectos de edificación de magnitud mediana a grande. Para cada proyecto en estudio, se revisó la incorporación de estos modelos para gestionar alguna de las 10 áreas de conocimiento propuestas por PMI. Además, se estudió en qué grupo de proceso (planificación, ejecución, y monitoreo y control) se utilizaron los modelos. El PMI presenta 47 procesos durante el ciclo de vida de un proyecto, estos son agrupados en 10 áreas de conocimiento y 5 grupos de procesos, 4 de los procesos están asociados al inicio y cierre de un proyecto, y los 43 restantes a los otros grupos, siendo estos la planificación, la ejecución y el monitoreo- control. Es por lo anterior, que sólo se analizarán los tres grupos anteriormente mencionados pues consideran la mayor cantidad de procesos y con ello la mayor parte del proyecto. Es importante mencionar que en los proyectos estudiados no necesariamente se explicita un alineamiento con los estándares del PMI, sino que se revisa la realización de los procesos que esta institución propone.

\section{USO DE LOS MODELOS ND EN LAS ÁREAS DE CONOCIMIENTO}

La guía de fundamentos para la dirección de proyectos (PMBOK) propone cuarenta y siete procesos agrupados en diez áreas de conocimiento que están vinculadas entre sí. En esta sección se relata el uso que se les ha dado a los modelos $\mathrm{nD}$, en las distintas áreas de conocimiento de dirección de proyectos, propuestas por el PMI.

\section{Alcance}

El alcance contiene procesos necesarios para asegurar que el proyecto incluya todo el trabajo requerido, y sólo el trabajo requerido, para completar el proyecto satisfactoriamente. Los procesos vinculados a esta 
área son: recopilar requisitos, definir el alcance, crear la estructura de desglose de trabajo (EDT), validar y controlar el alcance (PMI, 2013). Dentro de la revisión bibliográfica realizada, destacan los siguientes usos y/o impactos: BIM 4D se ha utilizado principalmente para apoyar la toma de decisiones en el diseño y planificación, además ha servido para definir correctamente el alcance de los proyectos. De igual manera se ha logrado identificar los beneficios y dificultades del modelado nD en los procesos de diseño y planificación de los sistemas de producción lo cual da cuenta de un alcance bien definido (Biotto et al., 2015). A partir de los modelos se pueden reconocer programas de construcción incompletos, identificando componentes del proyecto que no están o no tienen actividades asociadas en el programa (Mourgues y Fischer, 2001). Durante el proceso de diseño, en el modelo nD se pueden visualizar errores de procedimiento en la construcción del producto final o elementos que no corresponden con los requisitos del proyecto (en tiempo y forma). Lo anterior, se presenta en específico en el caso de estudio de un puente atirantado (Kim et al., 2011), lo que puede ser extrapolado a todo tipo de proyectos civiles.

Este tipo de modelos permite trabajar con múltiples niveles de detalles, según la necesidad que se requiera en una etapa específica de un proyecto (Kim et al., 2011; Russell et al., 2009). Además, entrega la posibilidad de ver actividades que necesitan mayor información a nivel del modelo de producto y programación (Mahalingam et al., 2010). También se puede utilizar para medir el progreso de la construcción, a partir del modelo 4D como línea base del alcance y con tecnología de sensores remotos (láser) para medir el avance real (Kim et al., 2013; Turkan et al., 2012).

\section{Tiempo}

La gestión del tiempo, incluye los procesos necesarios para lograr la conclusión del proyecto a tiempo, como la definición de actividades, definición de la secuencia entre actividades, estimación de recursos y duración de cada tarea, desarrollo del programa y control de éste (PMI, 2013). Dentro de la revisión bibliográfica realizada, se pueden destacar los siguientes usos y/o impactos en la gestión del tiempo:

Permite descubrir problemas de secuencias constructivas (Mourgues y Fischer, 2001), reduciendo significativamente la posibilidad de cometer errores y olvidar elementos de construcción, disminuyendo tiempos asociados a re-hacer trabajos (Porras et al., 2014). La programación basada en la ubicación anticipa conflictos de espacio- tiempo, mostrando componentes que son construidos en espacios de trabajo reducidos (Kala et al., 2010; Mourgues y Fischer, 2001; Su y Cai, 2014). Específicamente cuando se tienen que mover equipos y no se cuenta con los espacios requeridos para dicho proceso, por lo tanto, esta área permite planificar anticipadamente el espacio para el tránsito de maquinaria, camiones y/o movimiento de materiales (Mahalingam et al., 2010).

Visualiza la secuencia de la construcción, sobre todo cuando se trabaja desde varios frentes o cuando se quiere revisar en detalle trabajos particulares y/o peculiares en la industria (Mahalingam et al., 2010). Se generan escenas de construcción virtual con escalas apropiadas para que varios participantes puedan comunicarse y cooperar (Lian et al., 2016). Permite analizar las operaciones de construcción durante la fase de ejecución, simulando distintas estrategias de operación y coordinación entre contratistas (Hartmann et al., 2008). Se puede transparentar y optimizar el tiempo asignado en el programa para algunas tareas 0 conjunto de actividades específicas, a través de visualización y simulaciones de distintas alternativas y estrategias de construcción, antes de comenzar a ejecutar el proyecto (Mahalingam et al., 2010; Popov et al., 2010; Russell et al., 2009).

La inversión de la creación de un modelo de construcción paralela beneficia su constructividad e integra cantidades para programar la optimización (Kala et al., 2010). Incluso en el trabajo de Mahalingam et al. (2010), se valorizó la opinión de distintos actores en la industria de la AIC a través de una encuesta. Una de las hipótesis, fue que "los modelos 4D pueden ser usados en la fase de construcción, para optimizar la utilización de recursos, revisar los procesos del proyecto en orden de efectividad del plan y prevenir atrasos con seguimiento". Esta hipótesis fue valorizada con 4.03 en promedio (1 a 5) y una desviación estándar de 0.41. Permite definir el alcance de un equipo específico (por ejemplo, una grúa) y por lo mismo planificar las próximas actividades, asumiendo la restricción del alcance físico de este tipo de maquinaria (Mahalingam et al., 2010). Existen limitaciones en la modelación del tiempo, principalmente con los flujos de trabajo, dado que las tasas de producción y el flujo continuo de trabajo dependen obligatoriamente de otras herramientas que tienden a complejizar su modelación (Biotto et al., 2015).

\section{Costo}

La administración de los costos, incluyen los procesos involucrados en la planificación, estimación, preparación del presupuesto y control de costos de forma que el proyecto se pueda completar dentro del presupuesto aprobado (PMI, 2013). Dentro de la revisión bibliográfica realizada, destacan los siguientes 
usos y/o impactos de los modelos con estimaciones de costos: Se pueden hacer simulaciones para ahorrar materiales y recursos, para que luego puedan ser aplicados en la realidad. Además, se puede generar un sistema semi-automático para la evaluación de criterios económicos del diseño del proyecto, proporcionando al usuario la opción de verificar el gasto en cualquier etapa del proyecto (Popov et al., 2010), visualizando cantidades e integrando las mismas para programar los flujos de caja (Kala et al., 2010). En el trabajo de Lu et al., (2015) se propone un modelo de flujos de caja durante la ejecución del proyecto, basado en modelos virtuales y en el conocimiento de patrones de pago de materiales, equipo y mano de obra. El cálculo del presupuesto de construcción es más exacto que con el modelo tradicional (Porras et al., 2014). La poca masividad que ha habido en el uso de estos modelos se debe a que las empresas han centrado su análisis en la inversión inicial que conlleva su implementación y no en los impactos globales que genera (Ding et al., 2014). Sin embargo, se ha calculado que para ciertos proyectos el tiempo utilizado para realizar las estimaciones de costo se redujeron en un $25 \%$ en comparación a las metodologías tradicionales (Hartmann et al., 2008). Dentro de las hipótesis que valorizaron Mahalingam, et al., también estaba "la planificación, utilizando modelos 4D genera significativas ventajas desde el punto de la reducción de la cantidad de re-trabajos y costos extra, en comparación a la planificación tradicional". Esta hipótesis fue valorizada con 4.40 (1 a 5) en promedio y una desviación estándar de 0.26, siendo ésta la mejor valorizada por los distintos actores de la industria de la AIC (Mahalingam et al., 2010).

\section{Calidad}

La gestión de la calidad incluye todas las actividades de la organización ejecutante que determinan las políticas, los objetivos y las responsabilidades relativas a la calidad, de modo que el proyecto satisfaga las necesidades por las cuales se emprendió (PMI, 2013). Se reducen los errores de diseño significativamente, y sobre todo los relacionados con los procesos constructivos (Hartmann et al., 2008). Además, se puede utilizar para visualizar y revisar la constructividad de los distintos procesos. $Y$ por otro lado, se pueden revisar conflictos entre distintas especialidades (Mahalingam et al., 2010). La existencia de un sistema de gestión de la calidad facilita una mejor comprensión de la modelación en implementación de la producción, generando infraestructura de mejor estándar. Al aplicar normas de calidad, se ha demostrado que ello constituye un elemento determinante en la implementación de sistema de calidad, con impacto relevante al nivel de la adquisición de la capacidad organizacional y de gestión (Cruz, 2004).

\section{Riesgos}

La gestión de riesgos, incluye los procesos relacionados con la planificación de la gestión de riesgos, la identificación y el análisis de riesgos, las respuestas y el seguimiento y control de riesgos de un proyecto (PMI, 2013). El principal aporte a esta área en específico es que los modelos que consideran la dimensión del riesgo pueden mostrar problemas de accesibilidad y congestión dentro de la obra durante todo el proyecto (Mourgues y Fischer, 2001), con esta información se pueden prevenir los conflictos espaciotiempo (Chau et al., 2004; Su y Cai, 2014), para de esta manera ser capaces de formalizar las muchas incertidumbres que surgen durante las siguientes fases de la planificación (Mula et al., 2006). La clasificación y cuantificación de los riesgos se hace necesaria para tener una hoja de ruta con la cual mitigar los riesgos durante el desarrollo del proyecto; con ella es posible transformar información subjetiva y variables cualitativas a otras matemáticamente operables y con ello tener un monitoreo de la seguridad durante la construcción (Ding et al., 2014; Nieto-Morote y Ruz-Vila, 2011). En este sentido, existen otros sistemas basados en un análisis cuantitativo que considera criterios de tiempo, costo y condiciones de trabajo, los cuales delatan de forma gráfica (en colores) el nivel de riesgo en cada etapa del proyecto (Kang et al., 2013).

Los sistemas dan prioridad a condiciones peligrosas como factor relevante para el estudio del riesgo, dictaminando que dichas instancias se producen debido a la proximidad de los trabajadores, siendo necesaria la implementación de una matriz para la definición de distancias seguras entre los trabajadores, el uso de diagramas de tiempo y espacio (Dagan y Isaac, 2015). La congestión espacial temporal es posible cuantificarla en base a los indicadores DSI, que mide el riesgo en un trabajo local y entrega soluciones locales; y el CPI que permite tomar una ventaja de tiempo cualquiera para evaluar, analizar y comparar la acumulación de des-utilidad de cada entidad espacial (Chua et al., 2010).

\section{Comunicación}

El trabajo de comunicaciones, incluye todos los procesos necesarios para asegurar la generación, recogida, distribución, almacenamiento, recuperación y destino final de la información del proyecto en tiempo y forma (PMI, 2013). Los aportes de los modelos nD en esta materia son: (i) Aumenta la velocidad de respuesta a preguntas que se tienen del modelo, mejora la fluidez y rapidez de comunicación en terreno. Además, la cantidad de información requerida se reduce considerablemente (Mourgues et al., 2007). En la investigación 
de Hartman, et al., en un proyecto en particular las solicitudes de información fueron reducidas en un $80 \%$ y en otro en un 60\% (Hartmann et al., 2008); y (ii) Mejora en la comunicación entre el dueño, los diseñadores y el constructor, en comparación a los dibujos 2D (Mourgues et al., 2007). Realizando un análisis a diversas estructuras organizativas se puede apreciar que la cadena de suministros debe configurarse como una red dinámica o verdadera empresa virtual (Capó et al., 2007). Los modelos con varias dimensiones facilitan la creación de información y el compartir ésta en las etapas de diseño, construcción y mantenimiento de edificaciones, generando un ambiente de trabajo colaborativo (Popov et al., 2010). Otro aporte de los modelos nD es la facilidad de producción de elementos de documentación de diseño y construcción (Hartmann et al., 2008).

\section{Recursos humanos e Interesados}

La administración de los recursos humanos $(\mathrm{RRHH})$, incluye todos los procesos que organizan y dirigen al equipo de proyecto. Por otro lado, la gestión de los interesados, incluye todos los procesos para identificar, analizar, y gestionar a los stakeholders de un proyecto (PMI, 2013). La disposición de los recursos humanos es un factor relevante dentro de la optimización de los procesos en faena (Dagan y Isaac, 2015). Por ejemplo, cuando se tienen grandes cargas de trabajo es posible realizar un análisis multi-escala y asignar actividades precisas a todo el personal involucrado (Lian et al., 2016).

Dada la gran cantidad de stakeholders, los modelos nD resultan relevantes para la coordinación de las labores de los mismos, facilitando la comprensión y por tanto ejecución de las labores que cada uno de ellos debe realizar (Trebbe et al., 2015). Esto conlleva además un mayor involucramiento de los interesados, ya que existe una comunicación más sencilla y tangible, sobre todo con los interesados no técnicos (Dawood, 2010; Hartmann et al., 2008; Mourgues et al., 2007). Existe un mayor entusiasmo por parte de los trabajadores al tener que trabajar con modelos en las reuniones de coordinación. Por otra parte, esto conlleva a que haya un desafío de capacitación importante en lo que respecta a tecnologías de información en todo nivel en la industria de la AIC (Mourgues et al., 2007).

\section{Adquisiciones}

La gestión de adquisiciones, incluye todos los procesos para comprar o adquirir los productos, servicios o resultados necesarios fuera del equipo del proyecto para realizar el trabajo (PMI, 2013). Respecto a este punto se ha evidenciado que existe una necesidad de nuevos sistemas para la planificación y control de la producción que simultáneamente consideren tanto la planificación de los materiales como las capacidades, así optimizando el sistema de adquisiciones de la obra (El Ghazali et al., 2012; Mula et al., 2006). Se busca que mediante el sistema de escáner se pueda transferir la información ambiental real del sitio de trabajo rápidamente (Ding et al., 2014) y con un inventario en el sistema, se pueda recopilar y automatizar el proceso de compra de los recursos necesarios.

\section{Integración}

La integración, incluye procesos y actividades necesarios para identificar, definir, combinar, unificar y coordinar los distintos procesos y actividades dentro de los grupos de procesos de dirección de proyectos (PMI, 2013). Referente a ello, se han recopilado las siguientes ideas: Dada la existencia de múltiples contratistas en las obras de construcción se hace necesario proporcionar un sistema integrado, el cual evita la costosa improvisación in situ al proporcionar posibilidad de anticipar mejor los conflictos en la fase de planificación, integrando las actividades de las diversas cuadrillas permitiendo con ello la alineación de las actividades y la prevención de costosos problemas de coordinación (Trebbe et al., 2015). De esta forma es posible reducir esfuerzos y errores (evitar re-trabajos) para mejorar el desempeño en los proyectos (Feng et al., 2010).

Se ha logrado integrar elementos de alcance, tiempo y costo, que repercuten en otras áreas de conocimiento, vinculando automáticamente las distintas áreas y herramientas de la dirección de proyectos, sobre todo si se trabaja con modelos BIM (Feng et al., 2010). Además, estos modelos proporcionan escenas de construcción virtual, las cuales pueden ser visualizadas por todo el personal en tiempo real (Lian et al., 2016). El factor clave para que la integración sea efectiva y las técnicas cumplan con los beneficios esperados, es la continua actualización de los modelos, con el fin de tener información real y transparente para los distintos actores que la requieran, sobre todo cuando se asume el carácter dinámico representativo en la industria de la AIC (Kim et al., 2013; Kim et al., 2011; Lu et al., 2015; Mourgues et al., 2007).

\section{ANÁLISIS Y RESULTADOS}

La revisión bibliográfica evidenció la incorporación exitosa de los modelos nD en alguna de las 10 áreas de conocimiento de los 29 proyectos de edificación estudiados. La Fig. 1 muestra el porcentaje de proyectos donde se utilizaron modelos nD para gestionar cada una de estas las áreas de conocimiento propuestas por el PMBOK. 


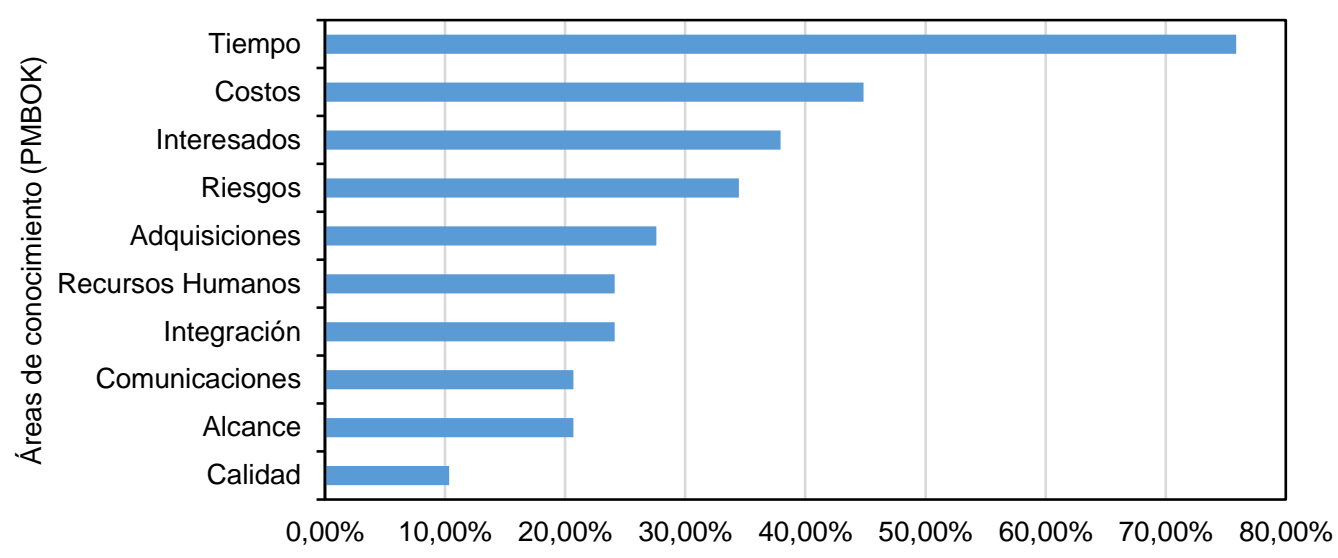

Porcentaje de proyectos donde se ha evidenciado su uso en un modelo $\mathrm{nD}$

Fig. 1: Evidencia del uso de modelos nD según área de conocimiento.

Se observa que un $80 \%$ de los proyectos consideró la dimensión del tiempo dentro de los modelos nD, quedando en segundo lugar la dimensión del costo, usada en un $44.83 \%$ ellos. La gestión de los interesados y del riesgo fueron consideradas en más del $30 \%$ de los casos. En menor medida, entre un $20 \%$ y $30 \%$ de los proyectos se evidenció la incorporación a los modelos $\mathrm{nD}$ de dimensiones ligadas a las adquisiciones, recursos humanos, integración y comunicaciones. El uso de la gestión de la calidad en modelos $\mathrm{nD}$ se observa solo en un $10.34 \%$ de los proyectos.

La Fig. 2 muestra en qué grupo de proceso se utiliza, en mayor o menor medida, cada una de las áreas de conocimiento en modelos nD. Se observa que, según grupo de proceso, existe una mayor aplicación de modelos $\mathrm{nD}$ durante la fase de planificación del proyecto (48\%), en segundo lugar, durante el monitoreo y control $(28 \%)$ y por último durante la fase de ejecución $(24 \%)$.

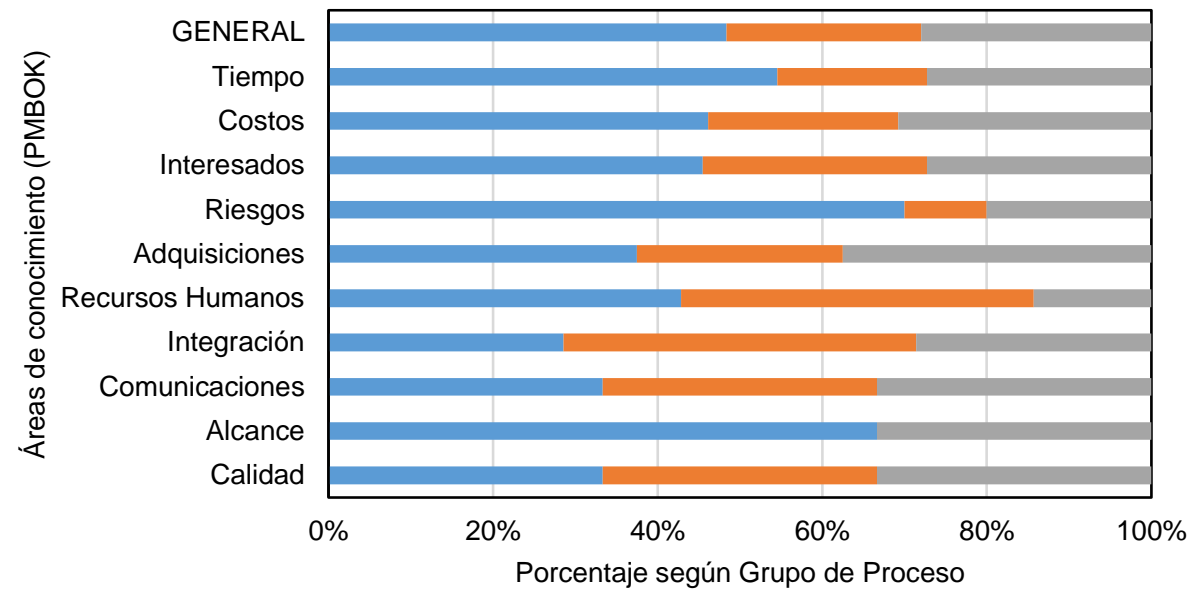

-PLANIFICACIÓN EJECUCIÓN MONITOREO Y CONTROL

Fig. 2: Distribución según grupo de proceso con respecto al total de evidencia por cada área de conocimiento.

Respecto a los 22 casos del uso del tiempo en modelos multidimensionales, se ha evidenciado que el $55 \%$ de ellos se ha desarrollado en la fase de planificación, lo que permite tener mayor claridad de las actividades que se realizarán, de la secuencia a considerar y de los tiempos asociados a las mismas. Esta consideración también tendrá efectos directos en las otras áreas de conocimiento, por ejemplo, la estimación del costo será más realista, los recursos humanos se planificarán en relación a los requerimientos de las actividades correspondientes a cada periodo, etc. Por otro lado, su uso en el proceso de ejecución ( $18 \%$ de las experiencias encontradas), y monitoreo y control ( $27 \%$ de las experiencias encontradas) vendrá dado por la reducción de los tiempos de espera (lo que influye en los recursos humanos y en las pérdidas) y el control del cronograma (procesos y flujos) respectivamente. De igual manera, el uso de la dimensión del costo se puede visualizar ampliamente en la fase de planificación (46\% de las experiencias encontradas), ligado a las estimaciones del presupuesto y en los otros procesos enfocados a disminuir las pérdidas y controlar los costos. 
El uso de modelos nD se ha enfocado ampliamente en la fase de planificación. De la evidencia obtenida un $45 \%$ de los casos relacionados con los interesados, un $70 \%$ de la evidencia ligada a la gestión del riesgo y un $67 \%$ de las experiencias $\mathrm{nD}$ relacionadas con el alcance, se enfocan en esta etapa. Estos números se explican dado que es en esta etapa inicial donde se busca optimizar la gestión de los interesados y la planificación de la misma con el fin de cumplir el alcance esperado en los plazos estimados. Se desea también poder definir planes para la prevención y respuesta ante riesgos posibles en etapas futuras. Además, es en esta etapa donde se considera que la recopilación de requisitos, la definición del alcance y la creación de las EDT, tendrán directa relación en la determinación de los costos, del tiempo, de los recursos humanos y las adquisiciones que deberán desarrollarse durante el proyecto.

La integración es un aspecto a considerar en los modelos nD. Se ha podido observar una mayor cantidad de evidencia en la etapa de ejecución (43\% de las experiencias encontradas), donde se busca gestionar el trabajo y alinear a los participantes de tal forma de optimizar sus rendimientos. Respecto a las comunicaciones, se evidencia un uso equitativo en todos los grupos de procesos, generando modelos que planifiquen los lineamientos comunicacionales, gestionen la comunicación para simplificar la comprensión en terreno y controlar la toma de decisiones. Estos factores tendrán directo impacto en la optimización de la variable Interesados. La revisión bibliográfica deja en evidencia lo poco usada que es la gestión de la calidad en modelos multidimensionales, no porque carezca de importancia, sino posiblemente por la mayor preponderancia que se ha dado a las otras áreas.

\section{CONCLUSIONES}

De acuerdo a los resultados de este estudio, y de su discusión y análisis se pueden extraer las siguientes conclusiones: 1) Los modelos nD tienen un gran potencial para lograr la preciada integración de los tres grandes componentes de la industria de la AIC, sobre todo cuando éstos tienden a ser bastante dinámicos y cualquier cambio que se realice en una de sus áreas impacta en todas las demás. Por este motivo los modelos nD logran servir como herramienta "columna vertebral" en el proyecto, recibiendo y entregando información entre otros modelos más específicos de cada área o especialidad. 2) Los modelos nD son claves en la integración de los diversos aspectos que posee la industria de la AIC, dado que logran conectar y modificar planes, diseños, insumos, personas, plazos, recursos, etc. Ello optimiza cada una de las labores y hace eficientes a los proyectos. Se ha evidenciado que el principal uso de los modelos nD está presente en la fase de planificación del proyecto, situación que es consistente con el impacto que una buena planificación tiene en el éxito de los mismos. 3) Para finalizar, se propone profundizar en la indagación de aún más evidencia respecto al uso de las metodologías nD, para validar cuantitativamente su uso masivo. Se propone también poder evaluar la integración simultánea del modelo nD en un mismo proyecto de la industria de la AIC, de tal forma de lograr la optimización máxima del mismo.

\section{AGRADECIMIENTOS}

Los autores agradecen a la Escuela de Ingeniería Civil de la Pontificia Universidad Católica de Valparaíso (Chile) y a la Vicerrectoría de Investigación de la Pontificia Universidad Católica de Chile que financia los estudios de postgrado de Rodrigo Herrera.

\section{REFERENCIAS}

Biotto, C. N., Formoso, C. T., e Isatto, E. L., O Uso da Modelagem BIM 4D no Projeto e Gestão de Sistemas de Produção em Empreendimentos de Construção, http://doi.org/10.1590/s1678-86212015000200015, Ambiente Construído, 15(1), 79-96 (2015)

Capó, J., Tomás, J. V., y Expósito, M., La gestión del conocimiento en la cadena de suministro: Análisis de la influencia del contexto organizativo, http://doi.org/10.4067/S0718-07642007000100017, Información Tecnológica, 18(1), 127-135 (2007)

Chau, K. W., Anson, M., y Zhang, J. P., Four-Dimensional Visualization of Construction Scheduling and Site Utilization, http://doi.org/10.1061/(ASCE)0733-9364(2004)130:4(598), Journal of Construction Engineering and Management, 130(4), 598-606 (2004)

Chua, D. K. H., Yeoh, K. W., y Song, Y., Quantification of Spatial Temporal Congestion in Four-Dimensional Computer-Aided Design, http://doi.org/10.1061/(ASCE)CO.1943-7862.0000166, Journal of Construction Engineering y Management, 136(6), 641-649 (2010)

Cruz, V. A., Sistema de Gestión de la Calidad en el Apoyo a la Implementación de Estrategias de Producción Ajustada, http://doi.org/10.4067/S0718-07642004000600010, Información Tecnológica, 15(6), 63-70 (2004) 
Dagan, D., e Isaac, S., Planning safe distances between workers on construction sites, http://doi.org/10.1016/j.autcon.2014.12.008, Automation in Construction, 50(C), 64-71 (2015)

Dawood, N., Development of 4D-based performance indicators in construction industry, http://doi.org/10.1108/09699981011024704, Engineering, Construction y Architectural Management, 17(2), 210-230 (2010)

Ding, L., Zhou, Y., y Akinci, B., Building Information Modeling (BIM) application framework: The process of expanding from 3D to computable nD, http://doi.org/10.1016/j.autcon.2014.04.009, Automation in Construction, 46, 82-93 (2014)

El Ghazali, Y., Lefebvre, É. y Lefebvre, L. A., The potential of RFID as an enabler of knowledge management and collaboration for the procurement cycle in the construction industry, http://doi.org/10.4067/S0718-27242012000400007, Journal of Technology Management and Innovation, 7(4), 81-102 (2012)

Feng, C. W., Chen, Y.J., y Huang, J. R., Using the MD CAD model to develop the time-cost integrated schedule for construction projects, http://doi.org/10.1016/j.autcon.2009.12.009, Automation in Construction, 19(3), 347-356 (2010)

Fulford, R. y Standing, C., Construction industry productivity and the potential for collaborative practice, http://doi.org/10.1016/j.ijproman.2013.05.007, International Journal of Project Management, 32(2), 315$326(2014)$

Hartmann, T., Gao, J. y Fischer, M., Areas of application for 3D and 4D models on construction projects, http://dx.doi.org/10.1061/(ASCE)0733-9364(2008)134:10(776), Journal of Construction Engineering and Management, 134, 776-785 (2008)

Kala, T., Seppänen, O., y Stein, C., Using an integrated 5D y location-based planning system in a large hospital construction project, Lean Construction Journal, 2010(July), 102-112 (2010)

Kang, L. S., Kim, S. K., Moon, H. S., y Kim, H. S, Development of a 4D object-based system for visualizing the risk information of construction projects, http://doi.org/10.1016/j.autcon.2012.11.038, Automation in Construction, 31, 186-203 (2013)

Kim, C., Kim, H., Park, T., y Kim, M. K., Applicability of 4D CAD in Civil Engineering Construction: Case Study of a Cable-Stayed Bridge Project, http://doi.org/10.1061/(ASCE)CP.1943-5487.0000074, Journal of Computing in Civil Engineering, 25(1), 98 (2011)

Kim, C., Son, H., y Kim, C., Automated construction progress measurement using a 4D building information model and 3D data, http://doi.org/10.1016/j.autcon.2012.11.041, Automation in Construction, 31, 75-82 (2013)

Lian, C., Lu, W., Rowlinson, S., y Zhang, X., Development of a Multifunctional BIM Maturity Model, J. Constr. Eng. Manage, 142 (11) (2016)

Lu, Q., Won, J., y Cheng, J.C.P., A financial decision making framework for construction projects based on 5D Building Information Modeling (BIM), http://doi.org/10.1016/j.jproman.2015.09.004, International Journal of Project Management, 34(1), 3-21 (2015)

Mahalingam, A., Kashyap, R., y Mahajan, C., An evaluation of the applicability of 4D CAD on construction projects, http://doi.org/10.1016/j.autcon.2009.11.015, Automation in Construction, 19(2), 148-159 (2010)

Mourgues, C., y Fischer, M., Investigaciones en Tecnologías de Información Aplicadas a la Industria A/E/C (Arquitectura, Ingeniería y Construcción), Center for Integrated Facility Engineering (2001)

Mourgues, C., Fischer, M., y Hudgens, D, Using 3D and 4D models to improve jobsite communication-virtual huddles case study, Proceedings of CIB $24^{\text {th }}$ W78 Conference $y 14^{\text {th }}$ EG-ICE Workshop y 5th ITC@ EDU Workshop, 91-97, Maribor, Slovenia, March (2007)

Mula, J., Poler, R., y García, J. P, Evaluación de Sistemas para la Planificación y Control de la Producción, http://doi.org/10.4067/S0718-07642006000100004, Información Tecnológica, 17(1), 19-34 (2006) 
Nieto-Morote, A., y Ruz-Vila, F., A fuzzy approach to construction project risk assessment, http://doi.org/10.1016/j.ijproman.2010.02.002, International J. Project Management, 29(2), 220-231 (2011)

Project Management Institute, A Guide to the Project Management Body of Knowledge (PMBOK® Guide), $5^{\text {a }}$ Ed., PMI Inc, Atlanta, U.S.A. (2013)

Popov, V., Juocevicius, V., Migilinskas, D., Ustinovichius, L., y Mikalauskas, S., The use of a virtual building design and construction model for developing an effective project concept in 5D environment, http://doi.org/10.1016/j.autcon.2009.12.005, Automation in Construction, 19(3), 357-367 (2010)

Porras, H., Giovanny, O., y Galvis, J., Elaboración De Modelos Del Proceso Constructivo 5D Con Tecnologías "Building Information Modeling " Methodology for Modelling the Construction Process 5D With Technologies Building Information, Revista Gerencia Tecnológica Informática, 14(38), 59-73 (2014)

Russell, A., Staub-French, S., Tran, N., y Wong, W., Visualizing high-rise building construction strategies using linear scheduling and 4D CAD, http://doi.org/10.1016/j.autcon.2008.08.001, Automation in Construction, 18(2), 219-236 (2009)

Su, X., y Cai, H., Enabling Construction 4D Topological Analysis for Effective Construction Planning, http://doi.org/10.1061/(ASCE)CP.1943-5487.0000463, J. Computing in Civil Engineering, 30(1),1-10 (2014)

Trebbe, M., Hartmann, T., y Dorée, A., 4D CAD models to support the coordination of construction activities between contractors, http://doi.org/10.1016/j.autcon.2014.10.002, Automation in Construction, 49(PA), 83-91 (2015)

Turkan, Y., Bosche, F., Haas, C. T., y Haas, R., Automated progress tracking using 4D schedule and 3D sensing technologies, http://doi.org/10.1016/j.autcon.2011.10.003, Automation in Construction, 22, 414-421 (2012) 
